\title{
DAW: Duplicate-AWare Federated Query Processing over the Web of Data
}

\author{
Muhammad Saleem ${ }^{1, \star}$, Axel-Cyrille Ngonga Ngomo ${ }^{1}$, Josiane Xavier Parreira $^{2}$, \\ Helena F. Deus ${ }^{2}$, and Manfred Hauswirth ${ }^{2}$ \\ 1 Universität Leipzig, IFI/AKSW, PO 100920, D-04009 Leipzig \\ lastname@informatik.uni-leipzig.de \\ 2 Digital Enterprise Research Institute, National University of Ireland, Galway \\ firstname.lastname@deri.org
}

\begin{abstract}
Over the last years the Web of Data has developed into a large compendium of interlinked data sets from multiple domains. Due to the decentralised architecture of this compendium, several of these datasets contain duplicated data. Yet, so far, only little attention has been paid to the effect of duplicated data on federated querying. This work presents DAW, a novel duplicate-aware approach to federated querying over the Web of Data. DAW is based on a combination of min-wise independent permutations and compact data summaries. It can be directly combined with existing federated query engines in order to achieve the same query recall values while querying fewer data sources. We extend three well-known federated query processing engines - DARQ, SPLENDID, and FedX - with DAW and compare our extensions with the original approaches. The comparison shows that DAW can greatly reduce the number of queries sent to the endpoints, while keeping high query recall values. Therefore, it can significantly improve the performance of federated query processing engines. Moreover, DAW provides a source selection mechanism that maximises the query recall, when the query processing is limited to a subset of the sources.
\end{abstract}

Keywords: federated query processing, SPARQL, min-wise independent permutations, Web of Data.

\section{Introduction}

The emergence of the Web of Data has resulted in a large compendium of interlinked datasets from multiple domains available on the Web. The central principles underlying the architecture of these datasets include the decentralized provision of data, the reuse of URIs and vocabularies, as well as the linking of knowledge bases [2. As a result, certain queries can only be answered by retrieving information from several data sources. This type of queries, called federated queries, are becoming increasingly popular within the Web of Data 1338912 14 2122]. Recently, the W3C released the SPARQL 1.1 specification which directly addresses federated queries 1. Due to the independence of

\footnotetext{
* This work was carried out while the author was a research assistant in DERI.

${ }^{1}$ http://www.w3.org/TR/sparql11-federated-query/
} 
the data sources, certain pieces of information (i.e., RDF triples) can be found in multiple data sources. For example, all triples from the DrugBank 2 and Neurocommons $\sqrt{3}$ datasets can also be found in the DERI health Care and Life Sciences Knowledge Bas屯4. We call triples that can be found in several knowledge bases duplicates.

While the importance of federated queries over the Web of Data has been stressed in previous work, the impact of duplicates has not yet received much attention. Recently, the work in [11] presented a benefit-based source selection strategy, where the benefit of a source is inversely proportional to the overlap between the source's data and the results already retrieved. The overlap is computed by comparing data summaries represented as Bloom filters [5]. The approach follows an "index-free" paradigm, and all the information about the sources is obtained at query time, for each triple pattern in the query.

In this paper we present DAW, a duplicate-aware approach for federated query processing over the Web of Data. Similar to [1] our approach uses sketches to estimate the overlap among sources. However, we adopt an "index-assisted" approach, where compact summaries of the sources are pre-computed and stored. DAW uses a combination of min-wise independent permutations (MIPs) [6] and triple selectivity information to estimate the overlap between the results of different sources. This information is used to rank the data sources, based on how many new query results are expected to be found. Sources that fall below a predefined threshold are discarded and not queried.

We extend three well-known federated query engines - DARQ 21, SPLENDID [8], and FedX [22] - with DAW, and compare these extensions with the original frameworks. The comparison shows that DAW requires fewer sources for each of the query's triple pattern, therefore improving query execution times. The impact on the query recall due to the overlap estimation was minimal, and in most cases the recall was not affected. Moreover, DAW provides a source selection mechanism that maximises the query recall when the query processing is limited to a subset of the sources.

The rest of this paper is zed as follows: Section 2 describes the state-of-the-art in federated query processing and different statistical synopsis approaches that can be used for approximating duplicate-free result sets. Section 3 describes our novel duplicate-aware federated query processing approach. An evaluation of DAW against existing federated query approaches is given in Section 4 . Finally, Section 5 concludes our paper and presents directions for future work.

\section{Related Work}

In recent years, many approaches have been proposed for federated query processing for the Web of Data. Quilitz and Leser [21] propose an index-assisted federated query engine named DARQ for remote RDF data sources.

\footnotetext{
2 http://datahub.io/dataset/fu-berlin-drugbank

3 http://neurocommons.org/page/RDF_distribution

4 http://hcls.deri.org:8080/openrdf-sesame/repositories/hclskb
} 
DARQ combines service descriptions, query rewriting mechanisms and a costbased optimisation approach to reduce the query processing time and the bandwidth usage. Langegger et al. [13] describe a solution similar to DARQ that relies on a mediator to keep its service descriptions up-to-date. SPLENDID [8 uses VOID 5 descriptions for data source selection along with SPARQL $A S K$ queries. All of the approaches described above can be considered to be index-assisted, since they all rely in some sort of local index to guide the source selection process. Index-free approaches include FedX [22] and the Avalanche system [3]. In FedX, the source selection is performed by using $A S K$ queries, while Avalanche gathers endpoints dataset statistics and bandwidth availability on the fly before the query federation. Ludwig and Tran 12 propose a hybrid query engine that assumes some incomplete knowledge about the sources to select and discover new sources at run time. A symmetric hash join is used to incrementally produce answers. Acosta et al. [1] present ANAPSID, a query engine that adapts the query execution schedulers to the SPARQL endpoints' data availability and run-time conditions.

Overlap estimation among data sources have been used in a number of approaches in the area of distributed and $\mathrm{P} 2 \mathrm{P}$ information retrieval 4 10 15 18 23 24. COSCO [10] gathers statistics about coverage and overlap from past queries and uses them to determine in which order the overlapping collections should be accessed to retrieve the most new results in the least number of collections. Bender et al. [4] describes a novelty estimator that uses Bloom filters [5] to estimate the overlap between P2P data sources. Bloom filters are also used in the BBQ strategy for benefit-based query routing over federated sources [1].

Statistical synopsis such as Min-Wise Independent Permutations (MIPs) 6], Bloom filters [5], Hash sketches [19], XSKETCH 20, fractional XSKETCH [7], and compressed Bloom filters [16] have been extensively used in the literature to provide a compacted representation of data sets. MIPs have been shown to be the provide a good tradeoff between estimation error and space requirements [15]6]. In addition, MIPs of different lengths can be compared, which can be beneficial for datasets of different sizes.

\section{Duplicate-Aware Federated Query Processing}

In this section we present our DAW approach. DAW can be used in combination with existing federated query processing systems to enable a duplicate-aware query execution.

Given a SPARQL query $q$, the first step is to perform a triple pattern-wise source selection, i.e., to identify the set of data sources that contain relevant results for each of the triple patterns of the query. This is done by the underlying federated system. For a given triple pattern, the relevant sources are also called capable sources. The idea of DAW federated query processing is, for each triple pattern and its set of capable sources, to (i) rank the sources based on how

${ }^{5}$ http://www.w3.org/TR/void/ 


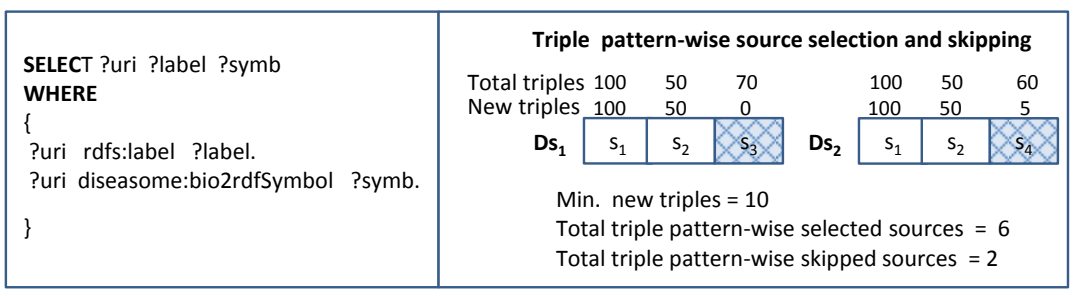

Fig. 1. Triple pattern-wise source selection and skipping example

much they can contribute with new query results, and (ii) skip sources which are ranked below a predefined threshold. We call these two steps triple patternwise source ranking and triple-pattern wise source skipping. After that, the query and the list of not skipped sources are forwarded to the underlying federated query engine. The engine generates the subqueries that are sent to the relevant SPARQL endpoints. The results of each subquery execution are then joined to generate the result set of $q$.

To better illustrate this, consider the example given in Figure 1, which shows a query with two triple patterns $\left(t p_{1}\right.$ and $\left.t p_{2}\right)$, and the lists of capable sources for both patterns. For each source we show the total number of triples containing the same predicate of the triple pattern and the estimated number of new triples, i.e. triples that do not overlap with the previous sources in the list. The triple pattern-wise source ranking step orders the sources based on their contribution. As we see in the example, for the triple pattern $t p_{1}$, source $S_{1}$ is ranked first, since it is estimated to produce 100 results. $S_{1}$ is followed by $S_{2}$, which can contribute with 40 new results, considering the overlap between the two sets. $S_{3}$ is ranked last, despite having more triples than $S_{2}$. This is because our duplicated-aware estimation could not find any triple in $S_{3}$ which is not in either $S_{1}$ or $S_{2}$. In the triple-pattern wise source skipping step, $S_{3}$ will be discarded, and $t p_{1}$ will not be sent to $S_{3}$ during query execution. We can also set a threshold on the minimum number of results. For instance, by setting the threshold to 10 results, source $S_{4}$ will be skipped, since it can only contribute with 5 new results for $t p_{2}$. By applying our duplicate-aware approach - which would select $S_{1}$ and $S_{2}$ both for tp1 and tp2 and would skip $S_{3}$ and $S_{4}$ - we would only send subqueries to two endpoints instead of four.

Both steps are performed prior to the query execution, by using only information contained in the DAW index. The main innovation behind DAW is to avoid querying sources which would lead to duplicated results. We achieve this by extending the idea of min-wise independent permutations (MIPs) [6], which are explained in the next section.

\subsection{Min-Wise Independent Permutations (MIPs)}

The main rationale behind MIPs is to enable the representation of large sets as vectors of smaller magnitude and to allow the estimation of a number of set 


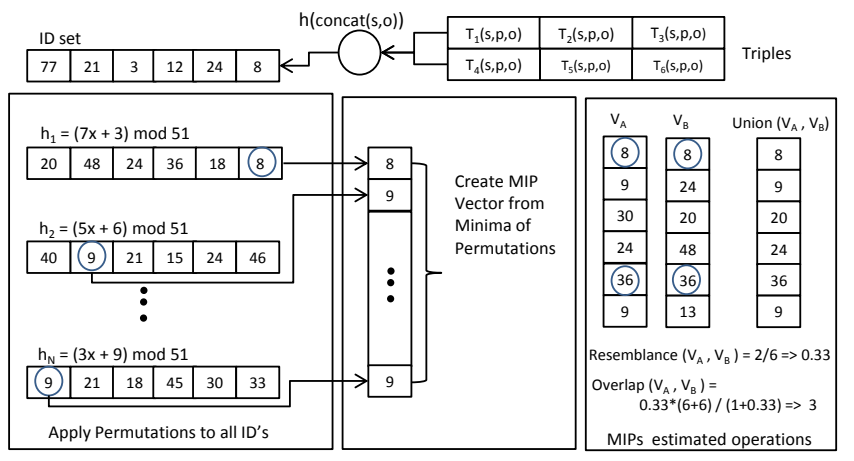

Fig. 2. Min-Wise Independent Permutations

operations, such as overlap and union, without having to compare the original sets directly. The basic assumption behind MIPs is that each element of an ordered set $S$ has the same probability of becoming the minimum element under a random permutation. MIPs assumes an ordered set $S$ as input and computes $N$ random permutations of the elements. Each permutation uses a linear hash function of the form $h_{i}(x):=a_{i}{ }^{*} x+b_{i} \bmod U$ where $U$ is a big prime number, $\mathrm{x}$ is a set element, and $a_{i}, b_{i}$ are fixed random numbers. By ordering the set of resulting hash values, we obtain a random permutation of the elements of $S$. For each of the $N$ permutations, the MIPs technique determines the minimum hash value and stores it in an $N$-dimensional vector, thus capturing the minimum set element under each of these random permutations. The technique is illustrated in Figure 2 ,

Let $V_{A}=\left[a_{1}, a_{2}, \ldots, a_{N}\right]$ and $V_{B}=\left[b_{1}, b_{2}, \ldots, b_{N}\right]$ be the two MIPs vectors representing two ordered ID's sets $S_{A}, S_{B}$, respectively. An unbiased estimate of the pair-wise resemblance between the two sets, i.e. the fraction of elements that both sets share with each other, is obtained by counting the number of positions in which the two MIPs vectors have the same number and dividing this by the number of permutations $N$ as shown in Equation 1 It can be shown that the expected error in the estimation $O(1 / \sqrt{N})$ [6]. Given the resemblance and the sizes of the two set, their overlap can be estimated as shown in Equation 2 , A MIPs vector representing the union of the two sets, $S_{A}$ and $S_{B}$, can be created directly from the individuals MIPs vectors, $V_{A}$ and $V_{B}$, by comparing the pairwise entries, and storing the minimum of the two values in the resulting union vector (see Figure 2). A nice property of MIPs is that unions can be computed even if the two MIPs vectors have different sizes, as long as they use the same sequence of hash functions for creating their permutations. In general, if two MIPs have different sizes, we can always use the smaller number of permutations as a common denominator. This incurs in a loss of accuracy in the result MIPs, but still yields to a more flexible setting, where the different collections do not have to agree on a predefined MIPs size [15.

$$
\operatorname{Resemblance}\left(S_{A}, S_{B}\right)=\frac{\left|S_{A} \cap S_{B}\right|}{\left|S_{A} \cup S_{B}\right|} \approx \frac{\left|V_{A} \cap V_{B}\right|}{N}
$$




$$
\operatorname{Overlap}\left(S_{A}, S_{B}\right) \approx \frac{\operatorname{Resemblance}\left(V_{A}, V_{B}\right) \times\left(\left|S_{A}\right|+\left|S_{B}\right|\right)}{\left(\operatorname{Resemblance}\left(V_{A}, V_{B}\right)+1\right)}
$$

In the DAW index, MIPs are used as follow: For a distinct predicate $p$ belonging to a data source $S$, we define $T(p, S)$ as the set of all triples in $S$ with predicate $p$. A MIPs vector is then created for every $T(p, S)$. First an $I D$ set is generated by mapping each triple in $T(p, S r)$ to an integer value. A triple is given in the form of subject, predicate and object tuples, i.e. $\langle s, p, o\rangle$. Since all triples in $T(p, S)$ share the same predicate by definition, the mapping is done by concatenating the subject $(s)$ and object $(o)$ of the triple, and applying a hash function to it (Figure 2). Then, the MIPs vector is created by computing the $N$ random permutations of each element in the $I D$ set and storing their minimum value. Finally, the MIPs vector is stored and mapped to each capability of the service description, as explained in the next section.

\subsection{DAW Index}

In order to detect duplicate-free subqueries, DAW relies on an index which contains the following information for every distinct predicate $p$ in a source $S$ :

1. The total number of triples $n_{S}(p)$ with the predicate $p$ in $S$.

2. The MIPs vector $M I P s_{S}(p)$ for the predicate $p$ in $S$, as described in the previous section.

3. The average subject selectivity of $p$ in $S, \operatorname{avgSbjSel}_{S}(p)$.

4. The average object selectivity of $p$ in $S$, avgObjSel ${ }_{S}(p)$.

The average subject and object selectivities are defined as the inverse of the number of distinct subjects and objects which appears with predicate $p$, respectively. For example, given the following set of triples:

$$
S=\left\{<s_{1}, p, o_{1}>,<s_{1}, p, o_{2}>,<s_{2}, p, o_{1}>,<s_{3}, p, o_{2}>\right\}
$$

the $\operatorname{avgSbjSel}_{S}(p)$ is equal to $\frac{1}{3}$ and the $\operatorname{avgObjSel}_{S}(p)$ is $\frac{1}{2}$. These two values are used in combination with the MIPs vector to address the expressivity of SPARQL queries as explained below.

Suppose that in a given triple pattern, neither the subject nor the predicate are bound. That means the pattern is of the form $\langle ? s, p, ? o\rangle$, where the question mark denotes a variable. In this case, the MIPs vectors in the DAW index can be used directly to estimate the overlap among the data sources that can provide results for the pattern. This is because the MIPs vectors are created by grouping triples according to their predicate. However, if any of the subject or object is bound (for example, $\left\langle s_{1}, p, ? o>\right.$ ), the selectivity of the pattern becomes much higher and the MIPs vectors alone are unable to address this. As a result, overlap will be overestimated. To address this issue the modify Equation 2 to account for the subject and object selectivities as follows:

$$
\operatorname{Overlap}_{t p}\left(S_{A}, S_{B}\right) \approx \frac{\operatorname{Resemblance}\left(V_{A}, V_{B}\right) \times\left(\left|S_{A}^{\prime}\right|+\left|S_{B}^{\prime}\right|\right)}{\left(\operatorname{Resemblance}\left(V_{A}, V_{B}\right)+1\right)}
$$


Listing 1.1. DAW index example

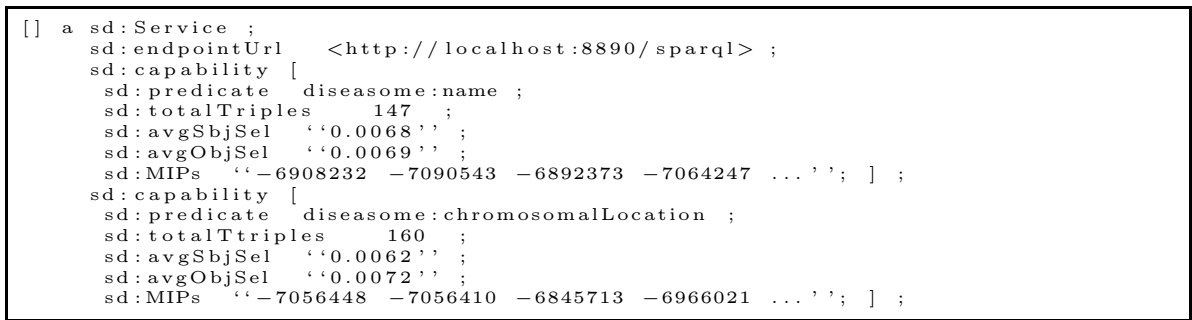

where the original size of a set $S_{i}$ is replaced by a value $\left|S_{i}^{\prime}\right|$ which is given by the following equation:

$$
\left|S_{i}^{\prime}\right|= \begin{cases}\left|S_{i}\right| & \text { if neither subject nor object are bound } \\ \left|S_{i}\right| \times \operatorname{avgSbjSel}_{S}(p) & \text { if subject is bound, } \\ \left|S_{i}\right| \times \operatorname{avgObjSel}_{S}(p) & \text { if object is bound. }\end{cases}
$$

We call the set $C_{S}(p)=\left\{p, n_{S}(p), \operatorname{avgSbjel}_{S}(p), \operatorname{avgObjSel}_{S}(p), \operatorname{MIPs}_{S}(p)\right\}$ a capability of the data source. The total number of capabilities of a data source is equal to the number of distinct predicates in it.

It is crucial to keep the index size small to minimise the pre-processing time. On the other hand, this index must also contain sufficient information to enable an accurate source selection and duplicate-free subquery generation. Some federated query approaches such as DARQ and SPLENDID already provide the total number of triples, as well as the average selectivity values. Therefore, the storage overhead create by the DAW index depends mostly on the size of the MIPs vectors which can be adjusted to any length. In general, MIPs can provide a good estimation of the overlap between sets with a few integer in length. An example of a DAW index is given in Listing [1.1.

\subsection{DAW Federated Query Processing}

As explained earlier, given a SPARQL query, DAW performs the triple patternwise source ranking and skipping steps in order to rank the sources based on how much they can contribute with new query results, and skip sources which are below a given threshold. In this section we describe these two steps in detail.

Triple Pattern-Wise Source Ranking: Given the heterogeneity and independence of data sources, it is expected that each source contributed differently in answering a given triple pattern, and the same result might be returned by multiple sources. Our goal is to provide a rank of the sources, according to the estimated number of new results it can contribute. By new results we mean with respect to the results already retrieved from sources ranked higher. 
The source ranking step works as follows: First, as no source has been ranked yet, the algorithm chooses the largest source, as it will likely to contribute with more results. To select the next source we use the DAW index to compute the estimated overlap between the already selected source and every remaining source. The remaining source with the least amount of overlap is then chosen and ranked second. Before selecting the next source in the rank, we first need to estimate the union of the already selected sources. This is needed since we want to find out how much a source can contribute with results are not in the sources selected so far. The union can be easily estimated by applying a vector operator on the original MIPs, as explained in Section 3.1. The new union MIPs can be further combined with other MIPs to get the estimation of the union among several sets. The source ranking step continues until no more sources are left to be ranked.

Triple Pattern-Wise Source Skipping: Given the rank of capable sources, the next step is to prune the rank, but skipping sources which cannot contribute with a minimum number of new results. This is done by setting a threshold, and pruning every source which falls below it. Since the total number of results depends on the triple pattern, the threshold is chosen in terms of the minimum percentage of new results a source can contribute. For instance, if the threshold is set to zero, DAW will aim at retrieving as much results as possible, while still skipping sources which cannot contribute with new results. Alternatively, the threshold can be set to higher values, in cases where the tradeoff between recall and number of sources queries is more important.

The pseudo code of the triple pattern-wise source ranking and skipping is given in Algorithm [1. It takes a triple pattern $t p_{i}(s, p, o)$, its list of capable sources $\mathbb{S}_{i}$, and the predefined threshold value as input and returned a ranked list of a subset of the capable source set $R_{i}, R_{i} \subseteq \mathbb{S}_{i}$ as output. The ranked list and the MPIs with the union of the selected sources are initialised with the largest source. Lines 8-14 adjust the size of the dataset to reflect the subject or object selectivities, depending on the query. Lines 15-16 estimate the overlap and number of new triples. The source with the highest amount of new triples is then selected (Lines 17-19). The triple pattern-wise source skipping is done in Line 23 and sources ranked higher than the threshold are added to the final ranked list (Line 24). The union MIPs is then updated (Line 26) and the algorithm continues until no more sources are left.

Before we present our experimental analysis of DAW it is important to note the difference between the number of triple pattern-wise sources and the number of sources (e.g. SPARQL endpoints). The total number of triple pattern-wise selected sources for a query is calculate as follow: Let $N S_{i} \in\{1 \ldots M\}$ be the number of sources capable of answering a triple pattern $t p_{i}$ where $M$ is the number of available (physical) sources. Then, for a query $q$ with $n$ triple patterns, $\left\{t p_{1}, t p_{2}, \ldots t p_{n}\right\}$, the total number of triple pattern-wise sources is the sum of the sources for individual triple patterns, i.e. $\sum_{j=1}^{n} N S_{j}$. In the example from Figure 1, the number of sources is $4\left(s_{1}, s_{2}, s_{3}, s_{4}\right)$ but the number of triple pattern-wise sources is equal to 6 . 


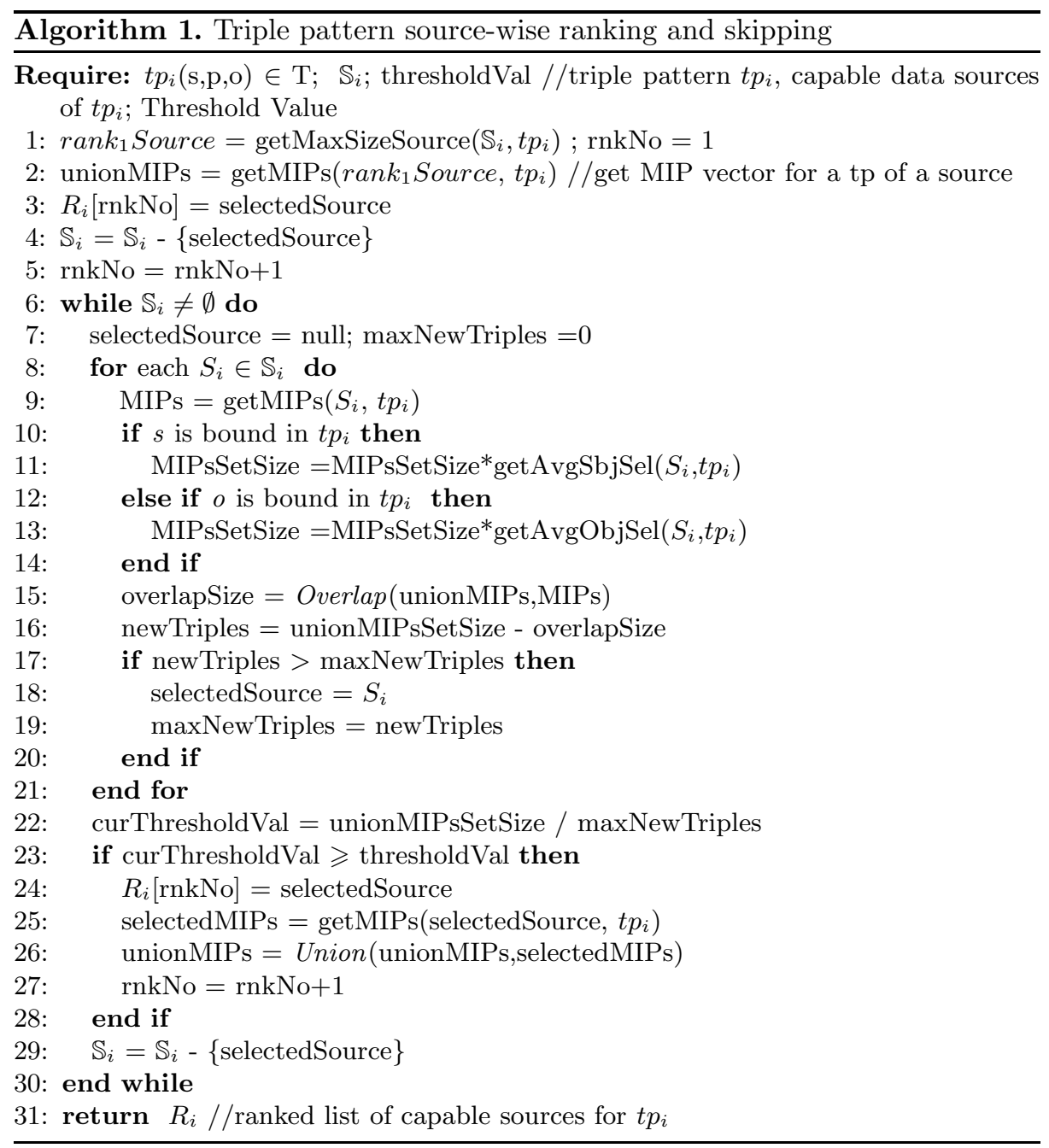

\section{Experimental Evaluation}

In this section we present an experimental evaluation of the DAW approach. We first describe the experimental setup, followed by the evaluation results. All data used in this evaluation can be found at the project web page 6

\subsection{Experimental Setup}

Datasets: For our experiments, we used four different datasets. The Diseasome dataset contains diseases and disease genes linked by disease-gene associations.

${ }^{6}$ https://sites.google.com/site/DAWfederation/ 
Table 1. Overview of the datasets used in the experiments

\begin{tabular}{|c|c|c|c|c|c|c|c|}
\hline Dataset & Number & Dataset & Index & Index. Gen. & Discrepancy & No. Duplicated & Duplicate \\
\hline & Triples & Size (MB) & Size (MB) & Time (sec) & & Slices & Slice ID \\
\hline Diseasome & 91,122 & 18.6 & 0.17 & 4 & 1,500 & 1 & 10 \\
\hline Publication & 234,405 & 39.0 & 0.24 & 6 & 2,500 & 1 & 10 \\
\hline Geo & $1,900,006$ & 274.1 & 1.63 & 133 & 50,000 & 2 & 5,8 \\
\hline Movie & $3,579,616$ & 448.9 & 1.66 & 201 & 100,000 & 1 & 2 \\
\hline
\end{tabular}

Table 2. SPARQL endpoints specifiTable 3. Distribution of query types cation across datasets

\begin{tabular}{lccc}
\hline EP & CPU(GHz) & RAM & Hard Disk \\
\hline 1 & $2.2, \mathrm{i} 3$ & $4 \mathrm{~GB}$ & $300 \mathrm{~GB}$ \\
2 & $2.9, \mathrm{i} 7$ & $16 \mathrm{~GB}$ & $256 \mathrm{~GB} \mathrm{SSD}$ \\
3 & $2.6, \mathrm{i} 5$ & $4 \mathrm{~GB}$ & $150 \mathrm{~GB}$ \\
4 & $2.53, \mathrm{i} 5$ & $4 \mathrm{~GB}$ & $300 \mathrm{~GB}$ \\
5 & $2.3, \mathrm{i} 5$ & $4 \mathrm{~GB}$ & $500 \mathrm{~GB}$ \\
6 & $2.53, \mathrm{i} 5$ & $4 \mathrm{~GB}$ & $300 \mathrm{~GB}$ \\
7 & $2.9, \mathrm{i} 7$ & $8 \mathrm{~GB}$ & $450 \mathrm{~GB}$ \\
8 & $2.6, \mathrm{i} 5$ & $8 \mathrm{~GB}$ & $400 \mathrm{~GB}$ \\
9 & $2.6, \mathrm{i} 5$ & $8 \mathrm{~GB}$ & $400 \mathrm{~GB}$ \\
10 & $2.9, \mathrm{i} 7$ & $16 \mathrm{~GB}$ & $500 \mathrm{~GB}$ \\
\hline
\end{tabular}

\begin{tabular}{lrrrrrrr}
\hline Dataset & STP & S-1 & S-2 & P-1 & P-2 & P-3 & Total \\
\hline Diseasome & 5 & 5 & 5 & 4 & 5 & 2 & 26 \\
Geo & 5 & 5 & 5 & - & - & - & 15 \\
Movie & 5 & - & - & - & - & - & 5 \\
Publication & 5 & 5 & 5 & 7 & 7 & 4 & 33 \\
\hline Total & 20 & 15 & 15 & 11 & 12 & 6 & 79 \\
\hline
\end{tabular}

The Publication dataset is the Semantic Web Dog Food dataset and contains information on publications, venues and authors of publications. The Geo dataset resulted from retrieving the portion of triples from DBpedia that maps resources to their geo-coordinates. Finally, the Movie dataset is the RDF version of IMDB and contains amongst others a large number of actors, movies and directors. To simulate a federated scenario with fragmented datasets distributed across several sources, we partitioned each dataset in 10 slices and distributed the slices across 10 data sources (one slice per data source). Each data source is a Virtuoso-201208-02 SPARQL endpoint with the specifications given in Table 2 .

To distribute the data across our 10 endpoints we defined a discrepancy factor, which controls the maximal size difference between the different slices.

$$
\text { discrepancy }=\max _{1 \leq i \leq M}\left|L_{i}\right|-\min _{1 \leq j \leq M}\left|L_{j}\right|
$$

where $L_{i}$ stands for the $\mathrm{i}^{\text {th }}$ slice. The data is first partitioned randomly among the slices in a way that $\sum_{i}\left|L_{i}\right|=D$ and $\forall i \forall j i \neq j \rightarrow|| L_{i}|-| L_{j}|| \leq$ discrepancy.

None of the existing benchmarks for federated query processing addresses the data duplication issue. Therefore, in order to add duplicates among slices, we randomly selected a number of slices and duplicated their contents across all remaining slices. For the DAW index, we use MIPs vectors of different sizes to better reflect the number of triples per predicate in each source. The sizes were chosen in a way that the overall index size is kept small. Table 1 presents an overview of the datasets, including the total number of triples and total size, the 
size of the DAW index, the index generation time, the discrepancy value among the 10 slices, the number of slices that were duplicated and their corresponding ID.

Queries: We used three types of queries in our experiments: Single triple patterns queries (STP), star-shaped queries (S-1, S-2), and path-shaped queries (P-1, P-2, P-3). Single triple pattern (STP) queries consist of exactly one triple pattern in the query. Star-shaped and path-shaped queries are defined as in [9]. A S-k star-shaped query has one variable as subject and $\mathrm{k}$ joins, i.e., $(\mathrm{k}+1)$ triple patterns. An example of a S-1 star-shaped query is given in Figure 1 A P-k pathshaped query is generated by using the object of one triple pattern as subject in the next triple pattern, and it also contains $(\mathrm{k}+1)$ triple patterns. Previous work has shown that these query shapes are the most common shapes found in real-world RDF queries [17. Our benchmark data consisted of 79 queries as shown in Table 3. Some query shapes could not be used on certain datasets due to the topology of the underlying ontology. For example, P-1 queries could not be sent to the Geo dataset since it only contained object properties. Each type a query was executed we used a random resource as subject or object, depending on the query type. The predicates of all queries are fixed.

Federated Query Engines: We implemented our DAW approach on top of three different federated query engines: DARQ [21, SPLENDID [8], and FedX [22]. Both DARQ and SPLENDID already provide an index with some of the statistics needed in DAW. Therefore, we only needed to extend this index. For FedX, which is indexfree, we added an index similar to the one in DARQ with our DAW extension. The underlying query execution mechanism remained the same.

Metrics: We compared the three federated approaches against their DAW extensions. For each query type we measured (i) the average number of triple pattern-wise sources that were skipped, (ii) the average recall, and (iii) the average query execution time. We did not consider the number of endpoints requests, as it depends on a number of factors, such as join type, block and buffer size, that vary across the different federated query processors. The threshold was initially set to zero, in order to maximise recall while querying fewer sources. All experiments were carried out in a machine with a $2.53 \mathrm{GHz}$ i5 processor, $4 \mathrm{~GB}$ RAM, and 500 GB hard disk. Experiments were carried out in a local network, so the network costs were negligible. After the first warm up run, each query type was executed 10 times and results were averaged.

\subsection{Experimental Results}

Triple Pattern-Wise Source Skipping: Table 4 shows the number of capable triple pattern-wise sources that were skipped by our approach, for each query type, as well as the recall. The total number of triple pattern-wise sources selected by the original systems is shown in brackets. The threshold was set to zero, which means that only sources that were estimated to returned no new 
Listing 1.2. A Single Triple Pattern (STP) query example

\section{SELECT ? title WHERE}

\{ www2008-paper:103 pub:title ?title.

Table 4. Distribution of the triple pattern-wise source skipped by DAW extensions for threshold value 0

\begin{tabular}{lcccccccc}
\hline Dataset & STP & S-1 & S-2 & P-1 & P-2 & P-3 & Total & Recall \\
\hline Diseasome & $14(35)$ & $30(77)$ & $40(107)$ & $35(65)$ & $65(125)$ & $30(50)$ & $214(459)$ & $100 \%$ \\
Geo & $22(40)$ & $23(55)$ & $37(101)$ & - & - & - & $82(196)$ & $99.99 \%$ \\
Movie & $22(38)$ & - & - & - & - & - & $22(38)$ & $100 \%$ \\
Publication & $9(30)$ & $10(37)$ & $15(86)$ & $14(60)$ & $21(120)$ & $32(102)$ & $101(435)$ & $100 \%$ \\
Total & $67(143)$ & $63(169)$ & $92(294)$ & $49(125)$ & $86(245)$ & $62(152)$ & $419(1128)$ & - \\
\hline
\end{tabular}

(a) DARQ

\begin{tabular}{lcccccccc}
\hline Dataset & STP & S-1 & S-2 & P-1 & P-2 & P-3 & Total & Recall \\
\hline Diseasome & $7(28)$ & $30(77)$ & $40(107)$ & $35(65)$ & $65(125)$ & $30(50)$ & $207(452)$ & $100 \%$ \\
Geo & $19(37)$ & $23(55)$ & $37(101)$ & - & - & - & $79(193)$ & $99.99 \%$ \\
Movie & $15(31)$ & - & - & - & - & - & $15(31)$ & $100 \%$ \\
Publication & $3(24)$ & $10(37)$ & $15(86)$ & $14(60)$ & $21(120)$ & $32(102)$ & $95(429)$ & $100 \%$ \\
Total & $44(120)$ & $63(169)$ & $92(294)$ & $49(125)$ & $86(245)$ & $62(152)$ & $396(1105)$ & - \\
\hline
\end{tabular}

(b) FedX and SPLENDID

results were pruned. We can see that DAW can effectively reduce the total triple pattern-wise selected sources, thus enable fewer subqueries federation. The highest gain was in the Diseasome dataset, where 214 sources were skipped in the DARQ approach, without affecting the recall. This corresponds to a decrease on the number of queried sources from 459 to 245 . In other words, a full recall was achieved by querying only $53 \%$ of the available triple pattern-wise sources. In all cases except in the Geo dataset, the recall was not affected and all relevant results were retrieved. In the Geo dataset, the DAW index incorrectly pruned a small number of relevant sources, but the recall was still $99.99 \%$. That means that DAW can deliver the same query results while querying much fewer sources. The source selection methods from FedX and SPLENDID return the same set of sources, therefore the number of skipped sources was the same for both. Moreover, they both use SPARQL ASK queries in the selection mechanisms, which leads to a better performance for STP queries. For example, consider the STP query given in Listing 1.2 where both the subject and predicate are bound. It is likely that a WWW2008 paper with id 103 is found in only one data source but the property pub:title may be found in every source. As a result, FedX and SPLENDID will only select a single capable source while DARQ will select all sources containing that predicate.

Query Execution Time: For each dataset and query type, we measured the average query execution time in each of the federated query approaches and also in their DAW extension. Again, the threshold was set to zero and the average was over 10 queries. Figures 3 , 4, and 5] show the results. We can see 


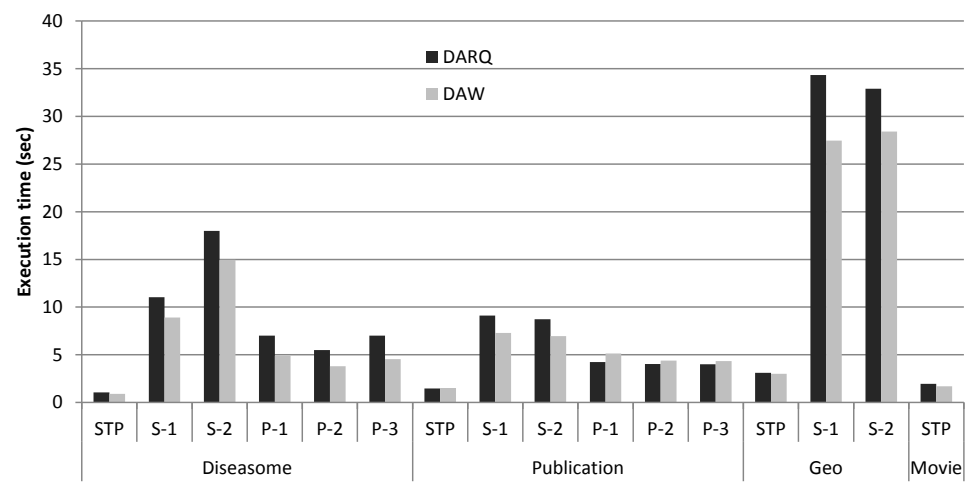

Fig. 3. Query execution time of DARQ and its DAW extension

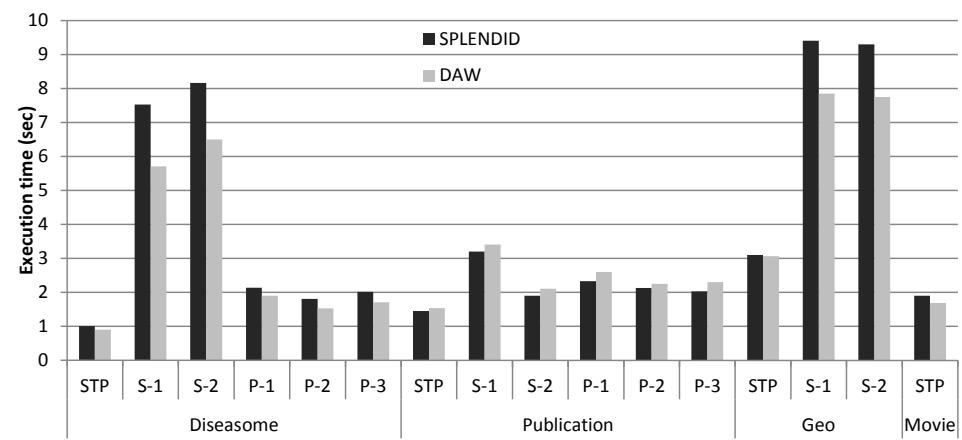

Fig. 4. Query execution time of SPLENDID and its DAW extension

that DAW improves the query performance for most of the cases. For three of the datasets, Diseasome, Geo and Movie, DAW improved the query execution times of all federated systems tested, for all query types. The query performance in the Diseasome dataset showed the highest improvements. This is due to the large number of triple pattern-wise sources that were pruned. We can also see that if the number of skipped sources is low - as for the Publication dataset - the overhead in computing the sources overlap can be higher than the execution time saved by querying fewer sources, so the overall query execution time is worse. The overall performance is summarised in Table 5 . We were able to improve the query execution time in DARQ by $16.46 \%$, the SPLENDID by $11.11 \%$, and FedX by $9.76 \%$. For the Diseasome dataset, the improvement for the DARQ approach was $23.34 \%$. These are averaged values across all datasets and query types. DAW led to a performance gain for most of the settings. We expect that in a setup with larger datasets and higher overlap, DAW can lead to even better improvements.

Number of Queried Sources vs. Query Recall: The evaluation presented so far focused on achieving full recall, and only discarded sources that the DAW index estimated to contribute with no new results. We have shown that the 


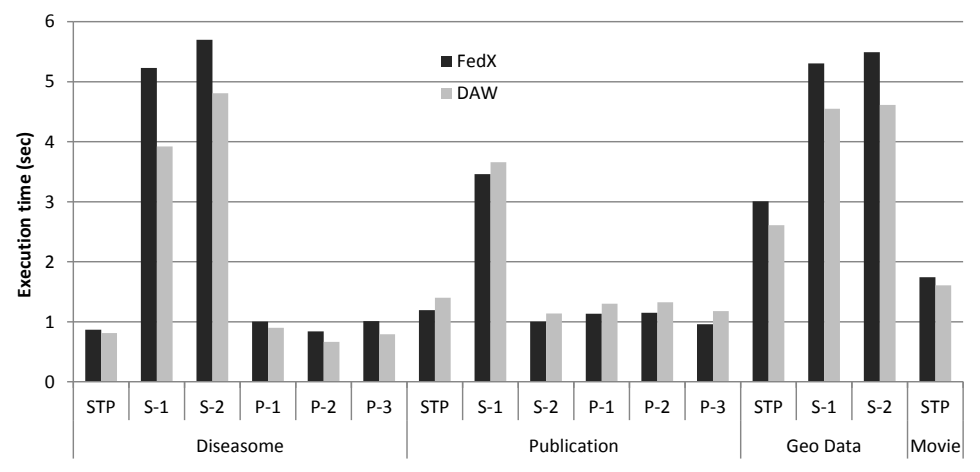

Fig. 5. Query execution time of FedX and its DAW extension

Table 5. Overall performance evaluation. Exe.time is the average execution time in seconds. Gain is the percentage in the performance improvement.

\begin{tabular}{|c|c|c|c|c|c|c|c|c|c|c|}
\hline & \multirow{2}{*}{\multicolumn{2}{|c|}{$\begin{array}{c}\text { Diseasome } \\
\text { Exe.time Gain }\end{array}$}} & \multicolumn{2}{|c|}{ Publication } & \multicolumn{2}{|c|}{ Geo Data } & \multicolumn{2}{|c|}{ Movie } & \multirow{2}{*}{\multicolumn{2}{|c|}{$\begin{array}{c}\text { Overall } \\
\text { Exe.time Gain }\end{array}$}} \\
\hline & & & Exe.time & Gain & Exe.time & Gain & Exe.tim & Gain & & \\
\hline$\overline{\mathrm{DA}}$ & 8.27 & & 5.26 & & 23.44 & & 1.96 & & 9.59 & \\
\hline & 6.34 & 23.34 & 4.94 & 6.14 & 19.62 & 6.31 & 1.68 & 13.88 & 8.01 & 6.46 \\
\hline PLENDID & 3 & & 2.18 & & 7.27 & & 1.90 & & 3.71 & \\
\hline & & 19.48 & & 3.94 & 6.22 & 14.40 & 1.68 & 11.16 & 3.30 & 11.11 \\
\hline & 2.44 & & 1.48 & & 4.6 & & 1.74 & & 2.44 & \\
\hline DAW & 1.98 & 18.79 & 1.67 & -12.38 & 3.92 & 14.71 & 1.61 & 7.59 & 2.20 & 9.76 \\
\hline
\end{tabular}

estimation given by our algorithm is quite accurate, as only $0.01 \%$ of the results in one dataset were missing. There might be cases, however, where full recall is not crucial and the query processing budget is limited. Here, the goal is to retrieve as many results as possible by querying only a subset of capable sources. Standard federated query processing approaches are only able to identify the set of capable sources. They are not able to compare the contribution of the sources in order to identify which subset yields to a better recall. With DAW, an approximation of this contribution is provided by the ranking step. For any given threshold, DAW is able to provide the subset of capable sources that will deliver the best recall for that number of sources. To demonstrate this, we computed the query recall for different threshold values for the DAW DARQ extension. We ran each of the STP queries 10 times on the Diseasome and Publication datasets and averaged the results. We varied the threshold value in order to limit the query to a fixed number of endpoints and we computed the query recall based on the DAW source selection. We compared it with the optimal duplicate-aware approach, where sources were manually selected to maximise the recall. The results are show in Figure 6. We can see that, in both cases, the source selection given by DAW is very close to the optimal case. Moreover, our experiment demonstrates the great potential in using source ranking for federated query processing. For the Diseasome dataset, by querying only 3 out of the 10 endpoints, DAW is able to retrieve $80 \%$ of the query results. A full recall is achieved with only 6 


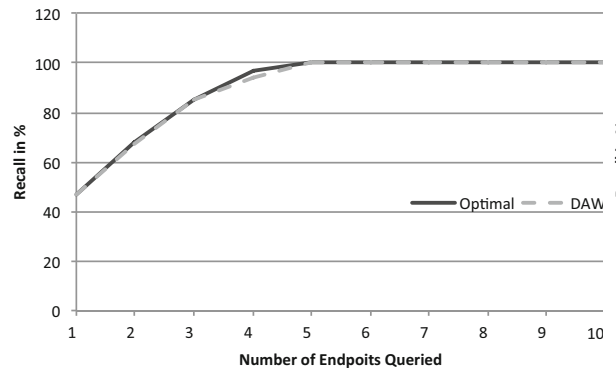

(a) Diseasome

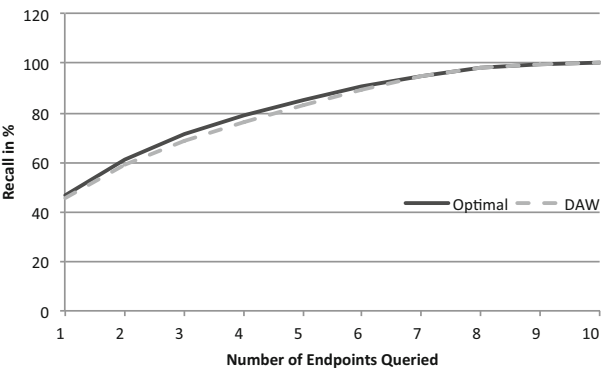

(b) Publication

Fig. 6. Recall for varied number of endpoints queried

endpoints. This naturally depends on the degree of overlap, but nevertheless it shows promising results that should be further explored.

\section{Conclusion and Future Work}

In this paper we presented DAW, an approach for duplicate-aware federated query over the Web of Data. DAW combines min-wise independent permutations with selectivity values to estimate the number of duplicate-free results. This estimation is used to first rank triple pattern-wise sources, based on their contribution, and to skip sources that contribute with little or no new results. DAW can be directly combined with existing index-assisted federated query processing systems, in order to improve the query execution. We evaluated our approach against DARQ, SPLENDID and FedX - three well known federated systems. The evaluation shows that by using the DAW extension the query execution times were improved in most of the cases, while recall was marginally affected. Moreover, DAW is suitable for maximising the recall for a fixed number of queried sources.

We will look at extending our index to further reduce the query execution time, for instance, by pre-computing some of the overlap statistics, based on query logs. The effect of different MIPs sizes and threshold values to find the optimal trade-off between execution time and recall will also explored, as well as different data partition methods.

Acknowledgments. This work has been supported by the European Commission under Contract No. FP720117287661 (GAMBAS), FP7-Granatum: RE7098, FP7-GeoKnow Grant No. 318159, by Science Foundation Ireland under Grant No. SFI/08/CE/I1380 (Lion-II) and Grant No. SFI/12/RC/2289 (INSIGHT).

\section{References}

1. Acosta, M., Vidal, M.-E., Lampo, T., Castillo, J., Ruckhaus, E.: Anapsid: an adaptive query processing engine for sparql endpoints. In: Aroyo, L., Welty, C., Alani, H., Taylor, J., Bernstein, A., Kagal, L., Noy, N., Blomqvist, E. (eds.) ISWC 2011, Part I. LNCS, vol. 7031, pp. 18-34. Springer, Heidelberg (2011) 
2. Auer, S., Lehmann, J., Ngonga Ngomo, A.-C.: Introduction to linked data and its lifecycle on the web. In: Rudolph, S., Gottlob, G., Horrocks, I., van Harmelen, F. (eds.) Reasoning Weg 2013. LNCS, vol. 8067, pp. 1-90. Springer, Heidelberg (2013)

3. Basca, C., Bernstein, A.: Avalanche: putting the spirit of the web back into semantic web querying. In: SSWS, pp. 64-79 (November 2010)

4. Bender, M., Michel, S., Triantafillou, P., Weikum, G., Zimmer, C.: Improving collection selection with overlap awareness in p2p search engines. In: SIGIR, pp. 67-74 (2005)

5. Bloom, B.H.: Space/time trade-offs in hash coding with allowable errors. Commun. ACM 13(7), 422-426 (1970)

6. Broder, A.Z., Charikar, M., Frieze, A.M., Mitzenmacher, M.: Min-wise independent permutations. IJCSS 60, 327-336 (1998)

7. Drukh, N., Polyzotis, N., Garofalakis, M., Matias, Y.: Fractional xsketch synopses for xml databases. In: Bellahsène, Z., Milo, T., Rys, M., Suciu, D., Unland, R. (eds.) XSym 2004. LNCS, vol. 3186, pp. 189-203. Springer, Heidelberg (2004)

8. Görlitz, O., Staab, S.: Splendid: Sparql endpoint federation exploiting void descriptions. In: COLD, ISWC (2011)

9. Harth, A., Hose, K., Karnstedt, M., Polleres, A., Sattler, K.-U., Umbrich, J.: Data summaries for on-demand queries over linked data. In: WWW, pp. 411-420 (2010)

10. Hernandez, T., Kambhampati, S.: Improving text collection selection with coverage and overlap statistics. In: WWW (Special interest tracks and posters), pp. 11281129 (2005)

11. Hose, K., Schenkel, R.: Towards benefit-based rdf source selection for sparql queries. In: SWIM, p. 2 (2012)

12. Ladwig, G., Tran, T.: Linked data query processing strategies. In: Patel-Schneider, P.F., Pan, Y., Hitzler, P., Mika, P., Zhang, L., Pan, J.Z., Horrocks, I., Glimm, B. (eds.) ISWC 2010, Part I. LNCS, vol. 6496, pp. 453-469. Springer, Heidelberg (2010)

13. Langegger, A., Wöß, W., Blöchl, M.: A semantic web middleware for virtual data integration on the web. In: Bechhofer, S., Hauswirth, M., Hoffmann, J., Koubarakis, M. (eds.) ESWC 2008. LNCS, vol. 5021, pp. 493-507. Springer, Heidelberg (2008)

14. Li, Y., Heflin, J.: Using reformulation trees to optimize queries over distributed heterogeneous sources. In: Patel-Schneider, P.F., Pan, Y., Hitzler, P., Mika, P., Zhang, L., Pan, J.Z., Horrocks, I., Glimm, B. (eds.) ISWC 2010, Part I. LNCS, vol. 6496, pp. 502-517. Springer, Heidelberg (2010)

15. Michel, S., Bender, M., Triantafillou, P., Weikum, G.: IQN routing: Integrating quality and novelty in $\mathrm{P} 2 \mathrm{P}$ querying and ranking. In: Ioannidis, Y., et al. (eds.) EDBT 2006. LNCS, vol. 3896, pp. 149-166. Springer, Heidelberg (2006)

16. Mitzenmacher, M.: Compressed bloom filters. IEEE/ACM Trans. Netw. 10(5), 604$612(2002)$

17. Morsey, M., Lehmann, J., Auer, S., Ngonga Ngomo, A.-C.: Dbpedia sparql benchmark: performance assessment with real queries on real data. In: Aroyo, L., Welty, C., Alani, H., Taylor, J., Bernstein, A., Kagal, L., Noy, N., Blomqvist, E. (eds.) ISWC 2011, Part I. LNCS, vol. 7031, pp. 454-469. Springer, Heidelberg (2011)

18. Nie, Z., Kambhampati, S., Hernandez, T.: Bibfinder/statminer: Effectively mining and using coverage and overlap statistics in data integration. In: VLDB, pp. 1097$1100(2003)$

19. Ntarmos, N., Triantafillou, P., Weikum, G.: Distributed hash sketches: Scalable, efficient, and accurate cardinality estimation for distributed multisets. ACM Trans. Comput. Syst., 27 (2009)

20. Polyzotis, N., Garofalakis, M.: Statistical synopses for graph-structured xml databases. In: SIGMOD, pp. 358-369 (2002) 
21. Quilitz, B., Leser, U.: Querying distributed rdf data sources with sparql. In: Bechhofer, S., Hauswirth, M., Hoffmann, J., Koubarakis, M. (eds.) ESWC 2008. LNCS, vol. 5021, pp. 524-538. Springer, Heidelberg (2008)

22. Schwarte, A., Haase, P., Hose, K., Schenkel, R., Schmidt, M.: Fedx: Optimization techniques for federated query processing on linked data. In: Aroyo, L., Welty, C., Alani, H., Taylor, J., Bernstein, A., Kagal, L., Noy, N., Blomqvist, E. (eds.) ISWC 2011, Part I. LNCS, vol. 7031, pp. 601-616. Springer, Heidelberg (2011)

23. Shokouhi, M., Zobel, J.: Federated text retrieval from uncooperative overlapped collections. In: SIGIR, pp. 495-502 (2007)

24. Si, L., Callan, J.P.: Relevant document distribution estimation method for resource selection. In: SIGIR, pp. 298-305 (2003) 